\title{
Phase Noise in the Photodetection of Ultrashort Optical Pulses
}

\author{
Jennifer A. Taylor ${ }^{+}$, Frank Quinlan*, Archita Hati*, \\ Craig Nelson*, Scott A. Diddams* \\ ${ }^{+}$Dept. of Physics, University of Colorado \\ Boulder, CO, USA \\ *Time and Frequency Division \\ National Institute of Standards and Technology \\ Boulder, CO, USA \\ Jennifer.Taylor@nist.gov
}

\author{
Shubhashish Datta, Abhay Joshi \\ Discovery Semiconductors, Inc. \\ Ewing, NJ, USA
}

\begin{abstract}
Femtosecond laser frequency combs provide an effective and efficient way to take an ultra-stable optical frequency reference and divide the signal down into the microwave region. In order to convert optical pulses into a usable RF signal, one must use high-speed photodetection; unfortunately, excess phase noise from both technical and fundamental sources can arise in the photodetection process. In order to ultimately minimize the noise effects of the photodetector, we must first characterize some of the known sources for noise arising in these devices. In this paper, we will study two sources of excess noise in high-speed photodiodespower-to-phase conversion and shot noise. The noise performance of each device will give us clues as to the nature of the sources, their effect on the output signal, and what design features of the photodiode minimize these noise effects.
\end{abstract}

\section{INTRODUCTION}

A self-referenced femtosecond laser frequency comb functioning as an optical-to-microwave divider can be a source of stable microwave signals possessing very low phase noise when stabilized to a high-finesse optical cavity [1-3]. Photodetection of the optical pulse train results in a corresponding train of current pulses, which in turn provides a comb of microwave frequencies at the laser repetition rate and its harmonics. Experiments have shown residual phase noise at levels below $-110 \mathrm{dBc} / \mathrm{Hz}$ for offsets of $1 \mathrm{~Hz}$ from the $10 \mathrm{GHz}$ harmonic, corresponding to instabilities at or below $1 \times 10^{-15}$ for $1 \mathrm{~s}$ averaging times [1-4].

These low levels of phase noise place stringent demands on the components of the optical and microwave systems, including the high-speed photodiodes used to convert the stable train of optical pulses into electronic microwave signals [2][5]. Previously, we have identified two of the sources of noise in the photodetection of ultrashort pulses to be the conversion of laser amplitude noise into electronic phase noise as well as the fundamental shot noise. The AMto-PM conversion arises in part due to saturation and other nonlinearities in the strongly driven photodiodes [6], while the shot noise provides the white noise floor.

In this paper, we compare the performance of a variety of photodiodes using a femtosecond frequency comb operating in the $900 \mathrm{~nm}$ spectral regime. We measure a $10 \mathrm{GHz}$ AM-toPM coefficient, defined as the induced RMS phase variation arising from a fractional optical power fluctuation $\left(\Delta \mathrm{P} / \mathrm{P}_{\mathrm{o}}\right)$. This coefficient is found to vary significantly among different photodiodes operating at different photocurrents, from $<0.1$ $\mathrm{rad} /\left(\Delta \mathrm{P} / \mathrm{P}_{\mathrm{o}}\right)$ to $>2 \mathrm{rad} /\left(\Delta \mathrm{P} / \mathrm{P}_{\mathrm{o}}\right)$. The coupled effect of photodiode saturation also leads to specific operating parameters for which the shot-noise limited noise floor is minimized. Again, this is found to vary significantly among different photodiodes, leading to the importance of such empirical measurements.

\section{TEST DIODES AND LASER}

We measure these noise effects using four different diodes with our laser system. The first photodiode, PD1, is a $22 \mathrm{GHz}$ top-illuminated InGaAs packaged photodiode. The diode area is coupled to an SMF, $\sim 9 \mu \mathrm{m}$ core pigtail. It is internally terminated at $50 \Omega$ and is externally biased at the manufacturer's recommended maximum of $7 \mathrm{~V}$. It is optimized for operation at $1550 \mathrm{~nm}$ but works over a wide spectral range. At $900 \mathrm{~nm}$, its responsivity is $0.3 \mathrm{~A} / \mathrm{W}$.

The second photodiode, PD2, is one of a pair of identical photodiodes housed in the same microwave package. It is a top-illuminated InGaAs packaged diode that employs an integrated graded-index (GRIN) lens at the end of its coupling fiber, SMF. The GRIN lens shapes the optical beam to produce a more uniform illumination profile (flat-top rather than Gaussian) on the photodiode; this suppresses peak photocurrent density and more effectively illuminates the 
whole diode [7][8]. This diode is internally terminated at 50 $\Omega$. It can be operated up to a peak bias voltage of $9 \mathrm{~V}$. The bandwidth is $22 \mathrm{GHz}$ at $1550 \mathrm{~nm}$. It also is optimized for $1550 \mathrm{~nm}$ but works over a wide spectral range. In the spectral range of $900 \mathrm{~nm}$, the responsivity is $0.26 \mathrm{~A} / \mathrm{W}$.

The third diode, PD3, is a packaged GaAs PIN detector with an SMF pigtail and an internal battery bias of $6 \mathrm{~V}$ and termination of $50 \Omega$. The fourth test photodiode, PD4, is one of another pair of similar photodiodes packaged together and coupled to SMF. It is a $12-\mathrm{GHz}$ top-illuminated InGaAs diode, but it features an InP cap layer of $0.3 \mu \mathrm{m}$ instead of 1.0 $\mu \mathrm{m}$ used for Photodiodes 1 and 2; Responsivity is $0.34 \mathrm{~A} / \mathrm{W}$ at $900 \mathrm{~nm}$. It is externally biased and operated at $9 \mathrm{~V}$. It is internally terminated at $50 \Omega$.

The optical signal is generated from a $1 \mathrm{GHz}$ Ti:Sapphire laser with a spectral peak in the region of $900 \mathrm{~nm}$, but with bandwidth extending down to $800 \mathrm{~nm}$. The pulse length at the end of a one meter SMF fiber is approximately $1 \mathrm{ps}$, as measured with an autocorrelator.

\section{SHOT NOISE}

Photodiodes convert incoming photons into an outgoing electrical current within the semiconductor media. There are intrinsic amplitude and phase fluctuations of the electrical current that arise from two fundamental phenomena: thermal noise and shot noise. Thermal (Johnson, resistive) noise is associated with random fluctuations of the current across resistive elements of the photodetection circuit (e.g., the load resistor of the photodiode). Shot noise is related to the randomness of the incident photon stream [9][10]. In a typical high-speed detection system with a $50 \Omega$ termination, the shot noise dominates the thermal noise for photocurrents greater than approximately $0.5 \mathrm{~mA}$ (see Figure 2(a)). It is this regime that we consider here.

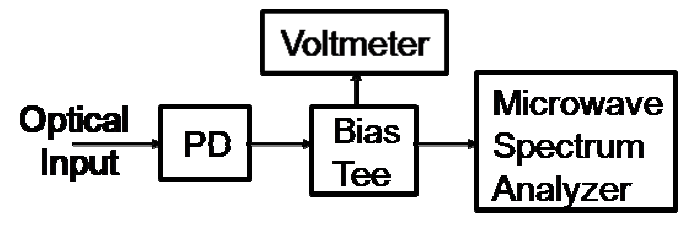

Figure 1. Schematic of shot noise measurement.

The rms shot noise current in a single quadrature (e.g., phase quadrature) is given by

$$
i_{\text {shot }}=\sqrt{e i_{\text {avg }} B W},
$$

where $\mathrm{BW}$ is the measurement resolution bandwidth (henceforth assumed to be $1 \mathrm{~Hz}$ ), $e$ is the elementary charge $\left(1.6 \times 10^{-19} \mathrm{C}\right)$, and $i_{\text {avg }}$ is the average photocurrent created by the incident optical power [9]. The shot noise power, $P_{\text {shot }}$, is related to the shot noise current by

$$
P_{\text {shot }}=i_{\text {shot }}^{2} R=e i_{a v g} R,
$$

where $R$ is the load resistor of the PD, typically $50 \Omega$ (Figure 2(a)). The thermal noise power at room temperature is shown for comparison as the dashed line in Figure 2(a). The spectrally white shot noise will be the fundamental noise floor in the detection of the microwave signals we are ultimately interested in.

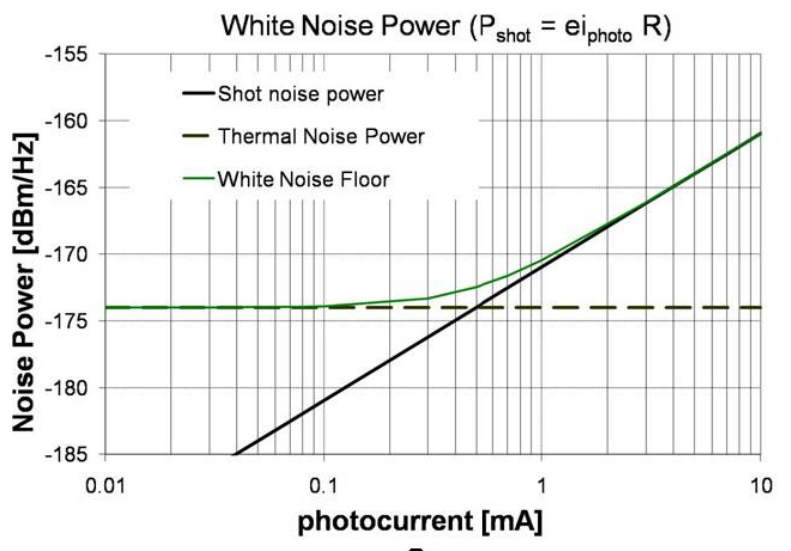

a.

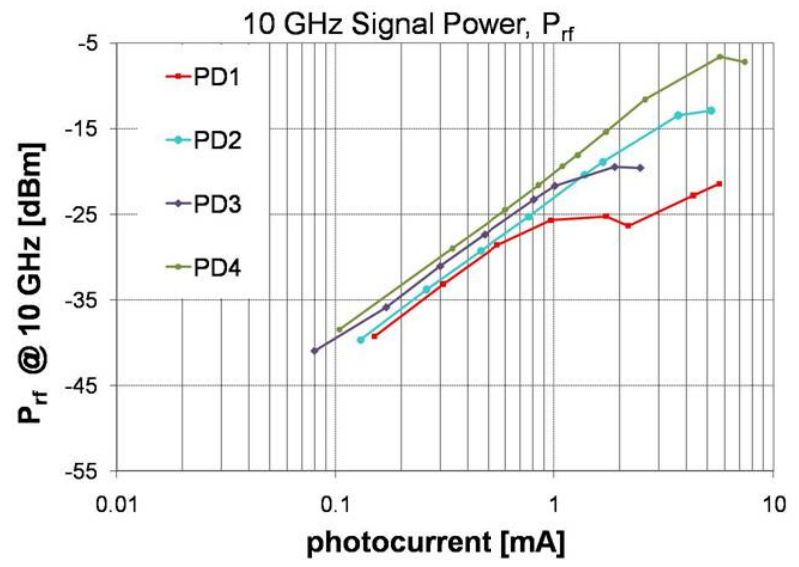

b.

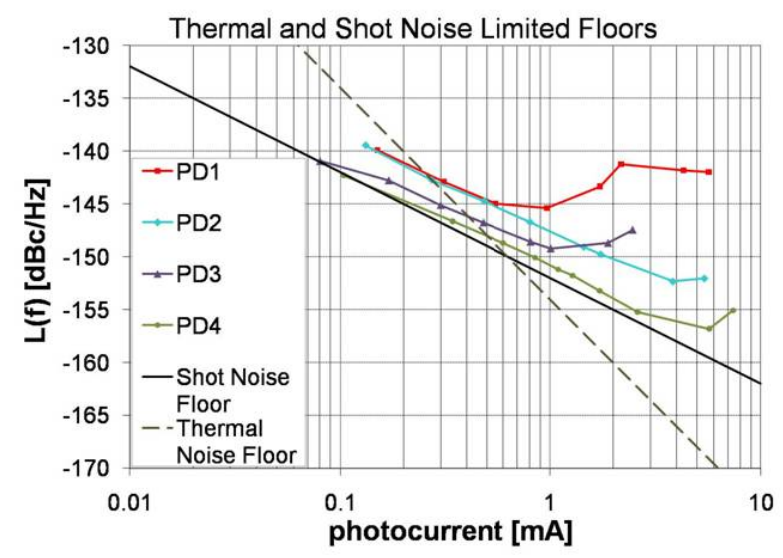

c.

Figure 2. a. Calculated thermal and shot noise power levels, and combined white noise level, for room temperature $(\mathrm{T}=300 \mathrm{~K})$. b. signal power for 10 $\mathrm{GHz}$ tone from laser pulse. c. Shot noise limited floor from measured hotocurrent, $i_{\text {avg }}$. Thermal limit shown for reference, but not included in calculation. 
We can determine the shot noise limited floor for the desired $10 \mathrm{GHz}$ signal by measuring the output of each PD on a microwave spectrum analyzer and on a voltmeter using a bias tee (see Figure 1). From the dc voltage reading, we can calculate average photocurrent simply using $i_{\text {avg }}=\mathrm{V} / \mathrm{R}$, where $\mathrm{R}=50 \Omega . P_{r f}$ at $10 \mathrm{GHz}$ is measured in units of $\mathrm{dBm}$ directly from the spectrum analyzer and is proportional to $i_{\text {avg }}^{2}$.

Projections of the shot noise limited floor, $L_{\phi}^{\text {shot }}$, as a function of average photocurrent were calculated from measured photocurrent for each photodiode and are plotted in Figure 2(c). Note, this plot does not include noise contributions from any other source, including thermal noise. The actual white noise floor (green plot in Figure 2(a)) can be measured, and we will present this analysis in future work. The shot noise floor for each PD varies slightly because each has different responsivity and saturation characteristics, resulting in different signal sizes at $10 \mathrm{GHz}$ [11]

In an actual measurement of the total noise floor, one would see a limitation from the thermal noise floor (dashed line in Figure 2(c)) until the point at which the shot noise exceeds the thermal noise, roughly $0.5 \mathrm{~mA}$ for a $\mathrm{PD}$ with responsivity of $0.3 \mathrm{~mA} / \mathrm{mW}$. PD1 and PD3 begin to saturate below $1 \mathrm{ma}$, and we see $L_{\phi}^{\text {shot }}$ increase as the photocurrent is increased. This is because the $10 \mathrm{GHz}$ signal power decreases under saturation, while the shot noise power continues to increase. PD2 and PD4 remain linear to about $2 \mathrm{~mA}$; PD2 appears to saturate at lower photocurrent than PD4. PD4 remains nearly linear up to $\sim 3 \mathrm{~mA}$, resulting in a projections of the noise limited power of below $-155 \mathrm{dBc} / \mathrm{Hz}$, representing a $10 \mathrm{~dB}$ improvement of the noise floor over PD1. The GRIN lens and thinner InP cap layer improve shot noise performance by improving the saturation limits of these diodes.

\section{AM-TO-PM CONVERSION}

Power-to-phase fluctuation is observed to adversely affect the transition from optical to microwave within the photodiode [5][11-13]. Broadening of a PD's electrical pulse (phase fluctuation) is observed when the incident optical power is increased (amplitude fluctuation). This means that there is a delay in the transmission of photocarriers generated by the incident pulse train across the photodiode. If more photocarriers are generated when the optical power is increased, then interactions between them generate internal electric fields opposing the bias field of the diode, increasing the transit time of a photocarrier and resulting in a broadening of the output pulse [14-17]. Ultimately, this affects the overall phase noise of the generated microwave signal. Increasing the bias voltage is one way of improving power-to-phase fluctuations; however, device failure due to runaway of dark current occurs when a bias voltage gets too large [8][14].

To quantify this effect for our four diodes, we define an AM-to-PM conversion as the induced RMS phase variation $(\Delta \varphi)$ arising from a fractional optical power fluctuation $\left(\Delta \mathrm{P} / \mathrm{P}_{\mathrm{o}}\right)$. We can directly measure " $\mathrm{rad} /\left(\Delta \mathrm{P} / \mathrm{P}_{\mathrm{o}}\right)$ " using a microwave phase bridge, shown in Figure 3 . We locked the repetition rate using a loop filter servo and a synthesizer referenced to a stable $10 \mathrm{MHz}$ maser. Then, an acousto-optic modulator (AOM) is used to insert a known, constant $20 \mathrm{kHz}$ modulation of about $1 \%$ modulation depth on the light source while also stepping the optical power into the PD using a neutral-density (ND) filter wheel. An rf mixer detects phase shifts due to power changes, and the output voltage is converted to radians using the mixer gain factor, $k_{d}[\mathrm{~V} / \mathrm{rad}]$. Since $k_{d}$ changes with signal power, we include a variable attenuator and amplifier combination in front of the mixer to compensate for changing optical power at the PD. Another 30 $\mathrm{kHz}$ amplitude modulation is placed on the test arm. This tone is monitored on the FFT analyzer and is minimized using the phase shifter. This sets quadrature at the mixer, allowing only phase modulation (PM) through to the mixer output.

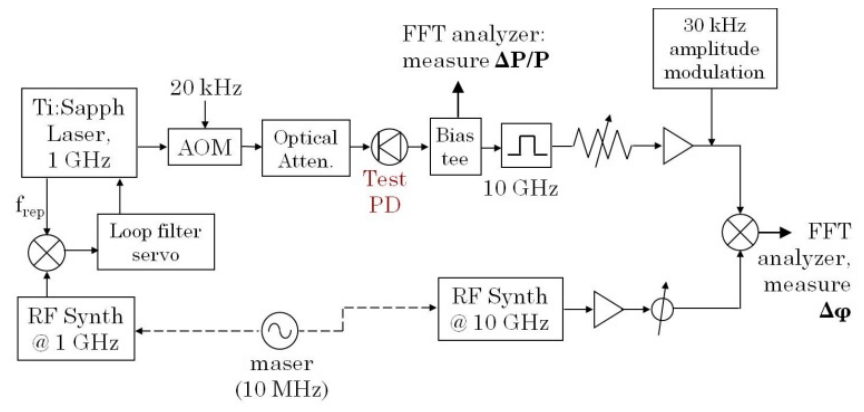

Figure 3. AM-to-PM conversion measurement system

The spectral power of the initial $20 \mathrm{kHz}$ amplitude modulation is measured on the FFT analyzer from the output of the bias tee and then divided by the optical power to find the normalized power of the input signal. The power in the 20 $\mathrm{kHz}$ tone from the output of the mixer (which contains only any phase modulation from AM-to-PM conversion in the PD) is then measured on the FFT analyzer and converted to radians, as described above. The ratio of the two gives us the power-to-phase conversion for this method.

Figure 4 shows the results of this measurement for the four diodes with varying optical power/photocurrent. PDs 1 and 2 have widely varying values, from as much as $2.3 \mathrm{rad} /\left(\Delta \mathrm{P} / \mathrm{P}_{\mathrm{o}}\right)$ to "nulls" where the AM-to-PM conversion factor drops to near zero, a phenomenon that has been previously observed [5]. The reason for the nulls is not completely understood; however, we have observed them to shift slightly depending on factors such as changes in external temperature, bias voltage, or type of optical fiber (resulting in changing mode shapes incident on the semiconductor material). In principle, one could tune the optical power to a null and operate there, but care must be taken-an unexpected shift could mean operating at a peak instead of a null.

PD3 also increases at higher photocurrent; however, it stays below $0.5 \mathrm{rad} /\left(\Delta \mathrm{P} / \mathrm{P}_{\mathrm{o}}\right)$ for a large range of lower photocurrents. $\mathrm{PD} 4$ is below $0.5 \mathrm{rad} /\left(\Delta \mathrm{P} / \mathrm{P}_{\mathrm{o}}\right)$ for the whole range of photocurrents that were measured. Using one of these diodes would be a practical way to lower amplitude-tophase conversion in general without requiring tuning to a sensitive null. 


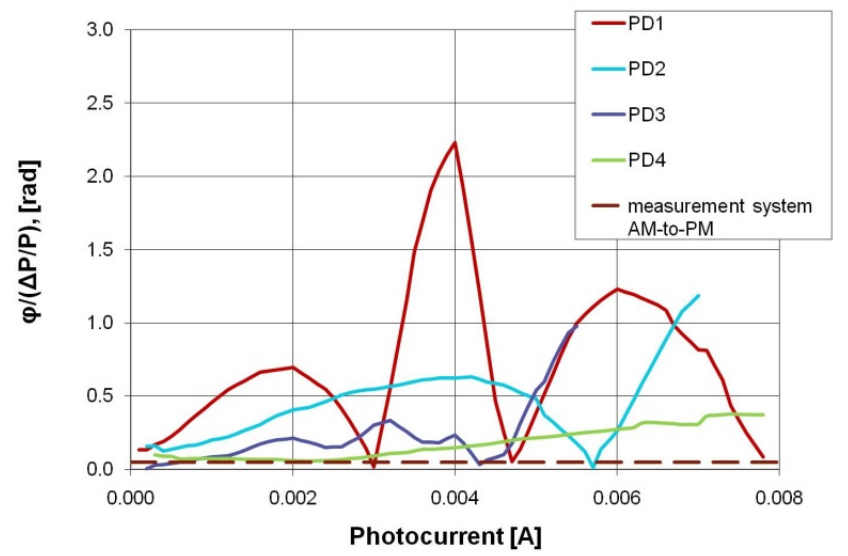

Figure 4. AM-to-PM conversion factor as a function of photocurrent (note that at $10 \mathrm{GHz}, 2 \pi$ corresponds to $100 \mathrm{ps}$ ).

Given an AM-to-PM conversion factor at a particular photocurrent and a known AM spectrum from our laser, we can estimate the phase noise by multiplying the spectrum by the conversion factor, as shown in Figure 5(a) and 5(b). We measured the AM spectrum of our Ti:Sapphire laser with the offset frequency unlocked, causing high AM in the $100 \mathrm{~Hz}$ to $1 \mathrm{kHz}$ region. The "worst case" AM-to-PM values for PD1 and PD2 are $2.3 \mathrm{rad}$ and $0.6 \mathrm{rad}$, respectively, at $4 \mathrm{~mA}$. To confirm these values, we did a residual phase noise measurement for each diode as well (Figure 5(c)). There is very good agreement between the estimate and the measurement in the high-AM region-PD1 gives just below $1 \times 10^{-4} \mathrm{rad} / \sqrt{\mathrm{Hz}}$ stability at the peak of its AM-to-PM conversion, while PD2 performs just above the $1 \times 10^{-5}$ $\mathrm{rad} / \sqrt{ } \mathrm{Hz}$ level at its maximum, which corresponds to a value of $\mathrm{L}(\mathrm{f}) \approx-100 \mathrm{dBc} / \mathrm{Hz}$. Even when operating in the "worst case" AM-to-PM regime, a good choice of photodetectors can reduce the ultimate phase noise.

\section{CONCLUSIONS AND FUtURE WORK}

We have discussed the limitations of shot noise and amplitude-to-phase conversion in photodiodes. As shown by the photodiodes tested here, the effect of these noise phenomena can vary depending on the diode used in the measurement system. A good choice of diodes in our system, for example, can result in a shot noise limited floor of less than $-155 \mathrm{dBc} / \mathrm{Hz}$ and a phase stability of $1 \times 10^{-5} \mathrm{rad} / \sqrt{\mathrm{Hz}}$, even at the least optimal photocurrent. Here we tested these diodes in the $900 \mathrm{~nm}$ spectral range; however, some diodes are optimized to work in the range of $1310 \mathrm{~nm}$ or $1550 \mathrm{~nm}$. Saturation properties can also change with different repetition rates or pulse shape. In future work, we would like to look at these diodes' performance with other combs in these regions. We would also like to compare the diodes in an absolute phase noise measurement between two Ti:Sapphire combs locked to different optical cavities to see if there is a significant change in noise level when using different diodes.

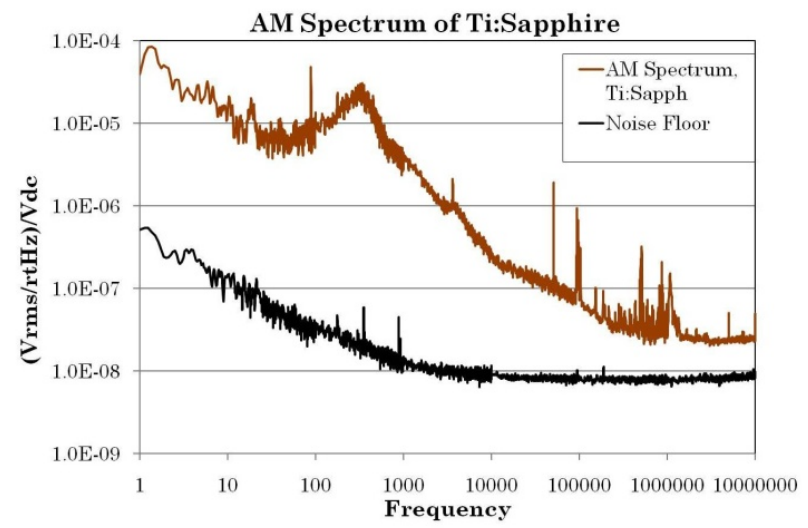

a.

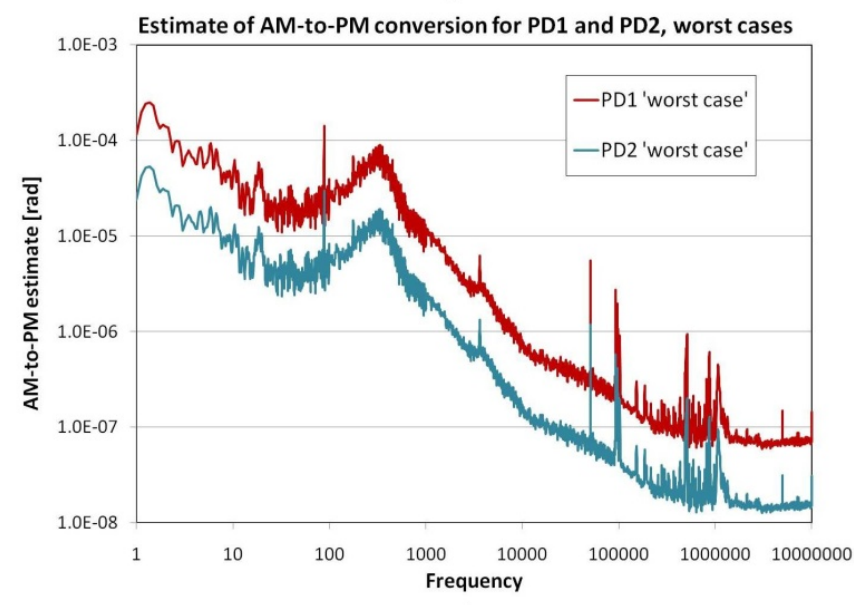

b.

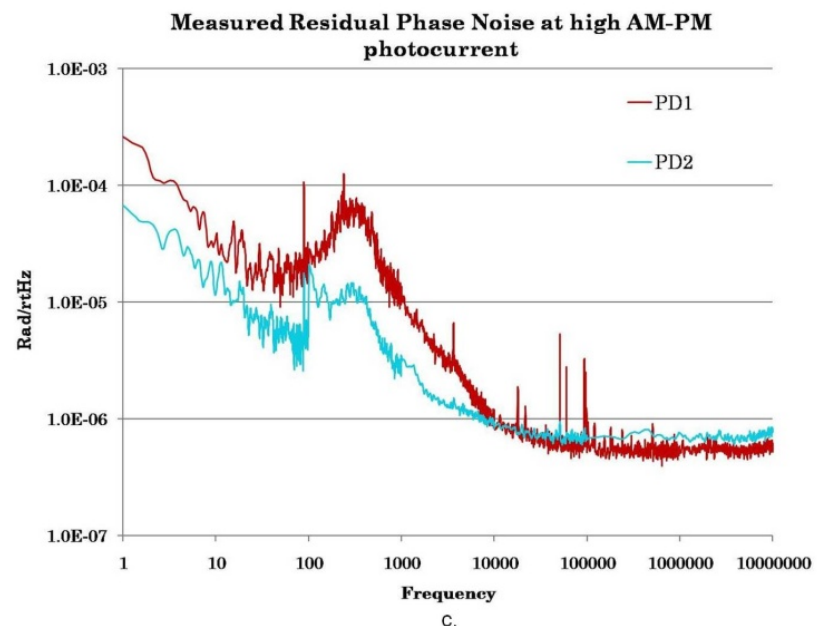

Figure 5. a. AM spectrum of $1 \mathrm{GHz}$ Ti:Sapphire laser. b. Estimate of phase noise from measured spectrum. c. Measured residual phase noise.

\section{ACKNOWLEDGMENT}

The authors thank Bill Swann and Todd Johnson for their contributions to this paper. 


\section{REFERENCES}

[1] A. Bartels, S.A. Diddams, C.W. Oates, G. Wilpers, J.C. Bergquist,W.H. Oskay, and L. Hollberg, "Femtosecond-laser-based synthesis of ultrastable microwave signals from optical frequency references," Opt. Lett. 30, 667-669 (2005).

[2] J.J. McFerran, E.N. Ivanov, A. Bartels, G. Wilpers, C.W. Oates, S.A. Diddams, L. Hollberg, "Low-noise synthesis of microwave signals from an optical source," Electron. Lett. 41, 36-37 (2006).

[3] Millo, J; Boudot, R; Lours, M; Bourgeois, P Y; Luiten, A N; Le Coq, Y; Kersalé, Y; Santarelli, G., "Ultra-low-noise microwave extraction from fiber-based optical frequency comb," Opt. Lett. 34, 3707-3709 (2009).

[4] W. Zhang, Z. Xu, M. Lours, R. Boudot, Y. Kersale, G. Santarelli, and Y. Le Coq, "Sub-100 attoseconds Optics-to-Microwave Synchronization," Applied Physics Letters (2010) (in press).

[5] E. Ivanov, S. Diddams, and L. Hollberg, "Study of the Excess Noise Associated with Demodulation of Ultra-Short Infrared Pulses, IEEE Trans. On UFFC, vol. 52, pp. 1068-1074, 2005.

[6] M. Currie and I. Vurgaftman, "Microwave Phase Retardation in Saturated InGaAs Photodetectors," IEEE Photonics Technology Letters, vol. 18, pp. 1433-1435, 2006.

[7] A. Joshi and D. Becker, "GRIN lens-couple top-illuminated photodetectors for high-power applications," in Proc. Microwave Photonics Conf., Victoria, Canada, Oct. 2007, Paper W-1.5

[8] A. Joshi, S. Datta, and D. Becker, "GRIN Lens Coupled TopIlluminated Highly Linear InGaAs Photodiodes," IEEE Photonics Technology Letters, vol. 20, pp. 1500-1502, 2008.
[9] Yariv, A, Optical Electronics, $4^{\text {th }}$ Edition, Saunders College Publishing, 1991, pp. 364-372.

[10] B.E.A. Saleh and M.C. Teich, Fundamentals of Photonics, John Wiley \& Sons, 1991, pp. 676-677.

[11] S. Foreman, K. Holman, D. Hudson, D. Johnes, and J.Ye, "Remote transfer of ultrastable frequency references via fiber networks," Review of Scientific Instruments, vol. 78, 021101 (2007).

[12] Characterization of Clocks and Oscillators: NIST Technical Note 1337, edited by D. Sullivan, D.Allan, D. Howe, and F. Walls, pp. 1925 (U.S. GPO, Washington, DC, 1990).

[13] E. Ivanov, S. Diddams, and L. Hollberg, "Analysis of Noise Mechanisms Limiting Frequency Stability of Microwave Signals," IEEE Journal of Selected Topics in Quantum Electronics, vol. 9, pp. 1059-1065, 2003.

[14] K. Williams, R. Esman, and M. Dagenais, "Nonlinearities in p-i-n microwave photodetectors," J. Lightwave Tech., vol. 14, no. 1, pp. 8496, Jan. 1996.

[15] K. Williams and R. Esman, "Observation of Photodetector Nonlinearities," Electronics Letters, vol. 28, pp. 731-732, April 1992.

[16] D. Tulchinsky and K. Williams, "Phase noise in compressed photodiodes," in MWP 2004 Tech. Dig., 2004, Paper WA-6, pp. 269272.

[17] M. Currie and I. Vurgaftman, "Microwave Phase Retardation in Saturated InGaAs Photodetectors," IEEE Photonics Technology Letters, vol. 18, pp. 1433-1435, 2006. 